Minireview

\title{
ABC lipid transporters: Extruders, flippases, or flopless activators?
}

\author{
Gerrit van Meer*, David Halter, Hein Sprong, Pentti Somerharju', Maarten R. Egmond \\ Membrane Enzymology, Bijvoet Center and Institute of Biomembranes, Utrecht University, Padualaan 8, \\ $3584 \mathrm{CH}$, Utrecht, The Netherlands
}

Received 22 November 2005; revised 6 December 2005; accepted 6 December 2005

Available online 19 December 2005

Edited by Ulf-Ingo Flügge

\begin{abstract}
Many mammalian ABC transporters move membrane lipids to acceptor lipid assemblies in the extracellular aqueous milieu. Because the desorption from the membrane costs more energy than provided by two ATPs, the transporter probably only translocates the lipid to a partially hydrophilic site on its extracellular face. From this high-energy site, the lipid may efficiently move to the acceptor, which ideally is bound to the transporter, or, in the absence of an acceptor, fall back into the membrane. If the lipid originated from the cytosolic membrane surface, this represents lipid flop and is probably a side activity of the transporters.
\end{abstract}

(c) 2005 Federation of European Biochemical Societies. Published by Elsevier B.V. All rights reserved.

Keywords: Flippase; Lipid asymmetry; Lipid translocation; Transbilayer movement

\section{ABC transporters and lipids}

A relation between mammalian $\mathrm{ABC}$ transporters and lipid transport was first found by Smit and colleagues [1]. When they knocked out the ABC transporter Mdr2 (Abcb4), a close relative of the prototype multidrug transporter MDR1 P-glycoprotein (ABCB1; [2]), they observed that the mice were jaundiced and found out that this was due to a lack of the phospholipid phosphatidylcholine (PC) in the bile. Around the same time, the accumulation of very longchain fatty acids in adrenoleukodystrophy was linked to deletions in ABCD1 [3]. Another milestone was the finding that even a multidrug transporter like ABCB1 is able to transport a wide variety of analogs of the regular membrane lipids across the plasma membrane [4]. The subsequent decade has witnessed the unmasking of many lipid-linked inherited diseases as being caused by mutations in one of the ABC transporters, and by now nearly half of the 49 or 50 human $\mathrm{ABC}$ transporters is thought to be involved in lipid transport $[5,6]$.

\footnotetext{
*Corresponding author. Fax: +31 302522478.

E-mail address: g.vanmeer@chem.uu.nl (G. van Meer).

${ }^{1}$ Permanent address: Institute of Biomedicine/Biochemistry, P.O. Box 63, Haartmaninkatu 8, Biomedicum, 00014 University of Helsinki, Finland.
}

\section{The dynamic organization of lipids in mammalian membranes}

A phospholipid bilayer is the basic structure of all membranes in mammalian cells. These membranes are not assembled de novo. They are created by fission from an existing membrane and, apart from mitochondria and peroxisomes, the cellular membranes continuously exchange proteins and lipids via the bidirectional traffic of membrane vesicles. Mammalian membranes have an oily consistency. Free diffusion of membrane components would predict that the continuous mixing would result in a uniform composition for all membranes. This is clearly not the case: the various organelles display different protein and lipid compositions, whereby it must be understood that they contain essentially the same lipids but in different ratios. The membranes of the late secretory pathway and the endocytotic pathway are enriched in sphingolipids and cholesterol, while in comparison the ER is highly enriched in unsaturated glycerophospholipids. According to the prevailing hypothesis, this is due to a spontaneous demixing of these lipids in the plane of the membrane. Preferential interactions with targeting proteins like SNARES must then ensure that the different lipid domains end up in separate transport carriers that ship them to their specific destinations [7].

The unique protein and lipid composition of each membrane endows them with unique physical properties. The membrane of the ER is highly fluid and, in addition, the phospholipid molecules rapidly equilibrate between the two leaflets of the lipid bilayer with a half-time of seconds. This property is lost upon the extraction and reconstitution of the ER lipids and for that reason the quest for the bidirectional ER lipid "flippase" is ongoing [8]. The evidence predicts that this activity is energyindependent. This is in contrast with, for example, the plasma membrane which displays an asymmetric arrangement of the various phospholipids, with an enrichment of the aminophospholipids phosphatidylserine (PS) and phosphatidylethanolamine (PE) in the cytosolic and the choline-containing phospholipids phosphatidylcholine (PC) and sphingomyelin (SM) in the exoplasmic bilayer leaflet. PS is virtually exclusively cytosolic and SM (and glycosphingolipids) exoplasmic. PE and PC have less pronounced asymmetric distributions. As in model membranes, the phospholipids do not freely move across the plasma membrane bilayer. Apart from that, the asymmetrical distribution is maintained by two mechanisms. First, SM and most glycosphingolipids are synthesized on the exoplasmic, lumenal aspect of the Golgi membrane, and, second, the aminophospholipids are actively "flipped" from exoplasmic to cytosolic surfaces by members of a subfamily of P-type 
A

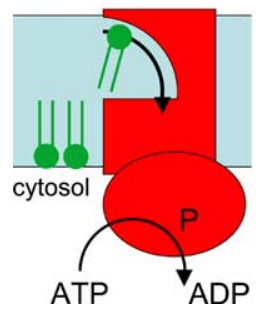

B

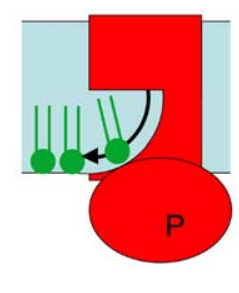

C

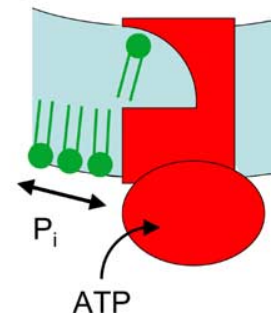

Fig. 1. P-type ATPases. P-type ATPases of the P4 subfamily have been suggested to "flip" specific phospholipid classes: they translocate the lipid from the exoplasmic leaflet (A) to the cytosolic leaflet of the plasma membrane (B) while inverting its headgroup orientation. Proteins of this family stimulate vesicle budding [10,11]. Probably, the shift of lipid mass across the membrane increases the lateral pressure in the cytosolic compared to the exoplasmic leaflet, which then relaxes by curving the membrane inwards (C), and promotes endocytosis.

ATPases. The subcellular location and substrate specificity of each of the 15 human members remain to be determined, but they are clearly important because a mutation in one of these results in hereditary cholestasis. Moreover, by moving lipid mass from one leaflet into the other they are thought to create a mass imbalance that may be one of the driving forces for the membrane bending (Fig. 1; [9]). In yeast, they play important roles in vesicle budding from the Golgi and endocytosis [10,11].

The rate by which a lipid spontaneously exchanges between the two bilayer leaflets is determined by its structure. Most membrane lipids, like the phospholipids and glycolipids, have headgroups of high polarity that reduce the rate of spontaneous transbilayer movement and typically yield half-times of hours for model membranes or plasma membranes like the erythrocyte membrane [11]. Neutralizing the headgroup charge by $\mathrm{pH}$ or by divalent cations can reduce the translocation halftime of, for example, free fatty acids [12] or phosphatidic acid from hours to seconds. Cholesterol with its sole hydroxyl moiety flips in seconds $[13,14]$ and this is also true for the second messenger lipids ceramide $(<1 \mathrm{~min})$ and diacylglycerol $(<1 \mathrm{~s}$; $[15,16])$. The difference in translocation half-times for these latter molecules demonstrates an effect of the lipid backbone as well. Translocation rates depend on the physical state of the membrane, and are higher in curved and loosely packed membranes.

\section{Monomeric lipid transport}

The bulk of the membrane lipids resides in membranes. This implies by no means that they are unable to leave the membrane. Each type of lipid partitions between the membrane and the aqueous phase. For most membrane lipids the monomeric solubility in water, which inversely correlates with molecular hydrophobicity and size, is exceedingly low. Still, some lipids do travel as monomers from the membrane to other acceptors with half-times that are relevant on biological time-scales. These half-times are essentially governed by the off-rate by which the lipid escapes from the membrane. The half-time of desorption is $\leqslant 100 \mathrm{~ms}$ for single chain lipids like fatty acids and lysophospholipids [17], 0.3-3 h for cholesterol, 4-80 h for long-chain phospholipids [18], and even longer for many sphingolipids [19]. As in the case of transbilayer translocation, the off-rate is modulated by the curvature and fluidity

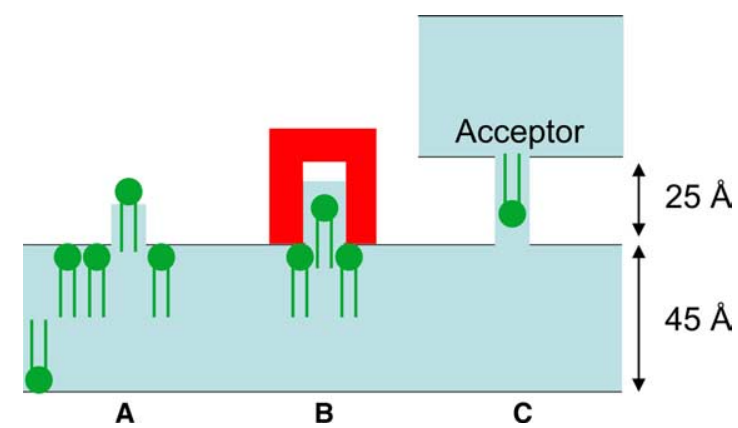

Fig. 2. (A) When a lipid desorbs from a membrane it goes through a thermal activation state, in which the contacts of its lipidic portion with the membrane have been broken $[18,20]$. The reversible partial projection of molecules out of the bilayer may precede their collisional capture by the acceptor [13]. (B) More likely, a transfer protein, or another low molecular weight acceptor like cyclodextrin, upon contact lowers the desorption activation energy by locally dehydrating the membrane [87]. (C) Also, the close proximity of an acceptor membrane (hydration repulsion starts at $25 \AA$ [88]), lipoprotein or micelle lowers the energy barrier during the collision (probably by ordering the water layer) thereby facilitating desorption [20,89].

of the membrane (up to two orders of magnitude faster from highly curved membranes and 10 times faster from unsaturated $\mathrm{PC}$ as compared to SM/cholesterol membranes, W.L.C. Vaz, personal communication). However, there is one factor intrinsic to the transport process itself that can dramatically speed up monomeric transport. Collisional contacts between the membranes or other lipidic structures can reduce the half-time of transport by orders of magnitude, probably by lowering the activation energy needed to overcome the aqueous barrier between donor and acceptor surface [20] (Fig. 2). It is not clear whether such collisions between lipid surfaces occur inside the cell, but, while ergosterol has been shown to equilibrate between ER and plasma membrane by non-vesicular transport with a half-time of 10-15 $\mathrm{min}$ [21], and therefore does not seem to require contact sites, stable contact sites are held responsible for the efficient transport of PC and PS from the ER to the mitochondria. An extreme example of facilitated desorption is the extraction of a lipid from a membrane by lipid-binding proteins like lipid transfer proteins. One of these, the ceramide transfer protein CERT, transports ceramide from the ER to the sphingomyelin synthase in the late Golgi [22]. CERT may actually be an active component of the contact sites between ER and Golgi [23]. Lipid transfer proteins of varying lipid specificity are present in the cell [7].

\section{ABC transporters and lipid desorption from cells}

The shedding of lipids by mammalian cells into the environment is a ubiquitous event. Often it represents the monomeric transport of a specific type of lipid from the plasma membrane to an acceptor (surface), and in nearly all cases, disorders involving defective lipid release from the plasma membrane have been correlated with mutations in an $\mathrm{ABC}$ transporter:

(a) Progressive familial intrahepatic cholestasis (PFIC). PFIC3: ABCB4 is required for the transport of PC from the bile canalicular membrane of hepatocytes onto bile micelles [1,24,25], but not the intestine [26]. PFIC2: The transfer of bile acids depends on the bile acid transporter 
ABCB11 [27,28]. Finally, cholesterol is exported by a heterodimer of the ABC half-transporters ABCG5 and G8, and depends on PC secretion [29].

(b) Sitosterolemia. ABCG5/G8 was also linked to the transport of nutritional (mostly plant) sterols back into the intestinal lumen [30].

(c) Tangier disease. The reverse transport of cholesterol onto lipid-poor apoA1 required ABCA1 [31-33], whereas ABCG1 and G4 are held responsible for transport of cholesterol onto high density lipoprotein (HDL) particles $[34,35]$.

In other cases, lipids are transferred into the lumen of organelles to be subsequently released by exocytosis or endocytotic recycling (see Fig. 3). Once again $\mathrm{ABC}$ transporter mutations have been correlated with disease:

(d) Fatal surfactant deficiency in newborns. ABCA3 [36] is required for transporting lipid molecules, mostly saturated PC, to the lung surfactant membranes filling the lamellar bodies in lung epithelial type II cells $[37,38]$. When released into the lung the surfactant spreads at the air-water interface in the alveoli.

(e) Harlequin ichthyosis. Similarly to ABCA3, ABCA12 is needed for the transport of lipids, mainly glucosylceramide, to the membranes within the lamellar bodies in keratinocytes [39-41]. These lamellar bodies fuse with the plasma membrane to release their contents in between the cells where the glucosylceramide is converted into the ceramide to form, together with fatty acids and cholesterol, the extracellular lamellar bilayers between the skin cells [42].

(f) X-linked adrenoleukodystrophy. This is a neurodegenerative disorder characterized by an accumulation of very long-chain fatty acids, caused by mutations in ABCD1, an $\mathrm{ABC}$ half-transporter. A subfamily of 4 ABC transporters (ABCD1 to ABCD4) has been related to the transport of these fatty acids or their SCoA esters across the peroxisomal membrane (see $[43,44]$ ).

(g) Stargardt macular dystrophy. An atypical situation exists for ABCA4. In the photoreceptor discs of the rod outer segments, ABCA4 seems to transport $N$-retinylidene-PE towards the cytosol $[45,46]$, which is the opposite direction of the other mammalian lipid-transporting ABC transporters. $N$-retinylidene-PE is released to the cytosol of the photoreceptors as all-trans-retinal, which is subsequently isomerized to 11-cis-retinal to be reutilized by the photoreceptor by a process that involves transport across the plasma membrane and extracellular space into the retinal pigment epithelial cells [47].

A subset of $\mathrm{ABC}$ transporters, the multidrug transporters and especially $\mathrm{ABCB} 1$ and $\mathrm{ABCC} 1$, exports a variety of drugs $[48,49]$ from cells. When expressed at high levels, these transporters are responsible for the chemoresistance phenotype. The substrates have in common that they are amphipathic, and they cover a wide range of hydrophobicity. Although they are generally extruded straight into the extracellular medium, some substrates are extruded efficiently only in the presence of an acceptor for small hydrophobic molecules like serum albumin. The fact that ABCB1 extrudes platelet activating factor (PAF), a short-chain ether $\mathrm{PC}$ [50,51], while its close relative ABCB4 transports regular long-chain PCs [1], shows that $\mathrm{ABC}$ transporters cover the whole range of hydrophobicity.

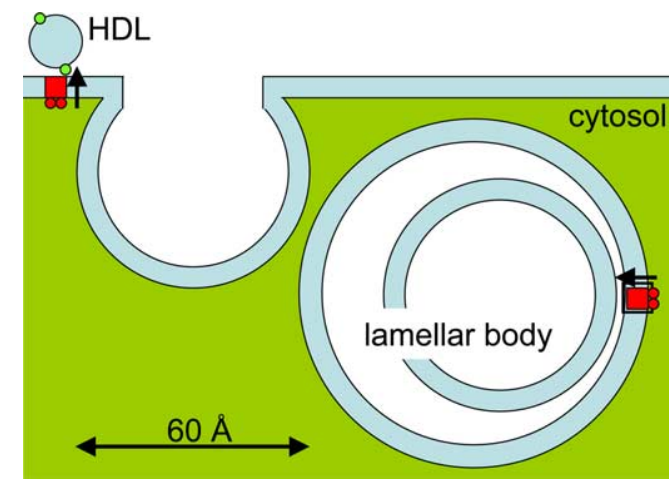

Fig. 3. ABC transporters (red) deposit (arrows) lipids into the surface monolayer of an extracellular acceptor like HDL, or into internal membranes inside the lamellar bodies, like the surfactant-containing storage organelles in lung type II cells or the glucosylceramidecontaining lamellar bodies in keratinocytes.

Finally, other mechanisms of lipid transport from the exoplasmic surface have been identified. The assembly of secretory lipoproteins in the lumen of the ER and Golgi cisternae requires the activity of the microsomal transfer protein MTP [52]. The loading of the immunoglobulin CD1b with a glycolipid in endosomes for presentation as an antigen was found to be mediated by the ATP-independent sphingolipid activator saposin $\mathrm{C}$ that normally presents glycosphingolipids to hydrolytic enzymes in the lysosomes [53].

\section{Mechanism of $\mathrm{ABC}$ transporter-assisted lipid export}

The available evidence suggests that most mammalian ABC transporters pick up their substrate lipid from the cytosolic surface. First of all, the multidrug transporters pump amphipathic substrates out of the cell, or into reconstituted vesicles, away from the side of ATP binding $[2,48]$. In addition, there is some evidence that natural phospholipids can be transported to the outside of the plasma membrane by an ABC transporter. The amount of newly synthesized, radiolabeled PC that could be depleted from the cell surface by the PC transfer protein or by a bile acid was higher in cells overexpressing ABCB4 $[54,55]$, but a role for vesicular traffic was not excluded in those studies. The exposure of PS on the surface of erythrocytes after the stimulation by a $\mathrm{Ca}^{2+}$ ionophore was reduced in ABCA1 null mice [56] and in a Scott patient with a missense mutation in ABCA1 [57]. Finally, the arrival on the erythrocyte surface of (radiolabeled) PC, newly synthesized on the inner leaflet of the membrane [58], was energy-dependent, sensitive to the $\mathrm{ABC}$ transporter inhibitor glibenclamide, and reduced in erythrocytes from Abcb1 null mice and from Abcb4 null mice [59].

In addition, a large body of experiments has shown that analogs of membrane lipids that carry one or two shortened (and often spin-labeled or fluorescent) fatty acyl chains can be translocated from the cytosolic surface by the $\mathrm{ABC}$ transporters $\mathrm{ABCA} 1, \mathrm{ABCB} 1, \mathrm{ABCB} 4$ or $\mathrm{ABCC} 1$ both in nucleated cells (e.g. [4,60-63] and erythrocytes [56,59,64,65], and these results are supported by studies on model membranes containing purified ABC transporters [66-68]. Although these reports still contain some unresolved contradictory results concerning the substrate specificity of the transporters, they all confirm the 
A
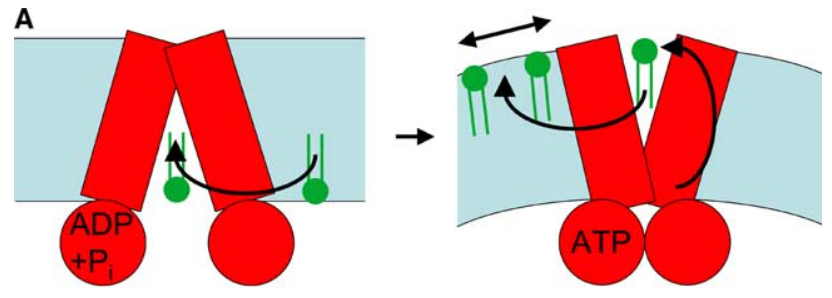

B

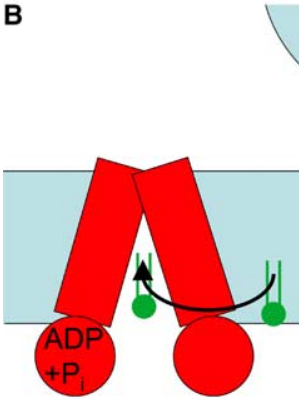

(1)

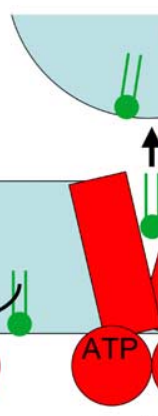

(2)
HDL

HDL

Fig. 4. Mechanistic models for ABC lipid transporters. (A) In the flippase model for ABC lipid transporters, the substrate lipid diffuses into the binding site on the cytosolic surface when the transporter is in the open conformation. ATP-binding dimerizes the nucleotide binding domains, closes the transporter, and flops the lipid. The lipid may be expelled during the resetting phase, generating a pressure increase in the exoplasmic leaflet. Alternatively, the lipid exchanges with a different lipid that has a higher affinity for the exoplasmic binding site. This lipid could then be flipped back, which would maintain the mass balance across the bilayer. (B) Alternatively, after the substrate enters the transporter (1), the conformational change may bring the lipid into an activated state without changing the lipid's orientation, hence: "flopless activation" $(2,3)$. If an acceptor is sufficiently close, for example if HDL were bound to the ABC transporter (2), the lipid could diffuse onto the acceptor. In the absence of an acceptor (3), the lipid may block the transporter. On the other hand, the lipid might relax from its energetically unfavorable position by entering the exoplasmic bilayer leaflet, thereby completing a "flop" movement. The lipid might change its orientation during or after the activation step.

binding of the substrate on the side of the ATP-binding domain, which is the cytosolic surface. The fact that also regular hydrophobic membrane lipids like PC [54,55,59], PS [56,57] and headgroup-labeled PE [67] enter the binding site predicts that the substrate can freely diffuse from the cytosolic bilayer leaflet into the binding site of the transporter.

Essentially, two models have been proposed for how $\mathrm{ABC}$ transporters extrude substrates from the cell. (i) In the flippase model (Fig. 4A; [69]), the substrate binds in the cytosolic half of the membrane, is translocated during the ATP-consuming conformational change of the transporter with a reversal of its orientation, followed by release into the exoplasmic half of the bilayer. After this "flop" movement the molecule is free to partition between the membrane and the extracellular medium. Relatively hydrophilic drugs would quickly partition into the external environment. However, this model offers no explanation for how lipids reach extracellular acceptors and why this requires the transporter. (ii) The hydrophobic "vacuum cleaner" model predicts that the substrate enters a binding site in the membrane and is subsequently ejected into the extracellular medium [70]. This mechanism thus involves the complete hydration of the substrate molecule upon the conformational change of the transporter. This poses a problem in the case of the regular membrane lipids: the Gibbs' molar free energy

of complete desorption from the membrane into an aqueous phase for a phospholipid is some $100 \mathrm{~kJ} / \mathrm{mol}$ [18], whereas ATP has an energy content of $30 \mathrm{~kJ} / \mathrm{mol}$. Assuming that the ABC transporters consume one or two ATP during their "power stroke", this would generate insufficient energy for the extrusion of a phospholipid molecule.

A third, "activation", model offers a solution for the incongruencies of the models above (Fig. 4B). Instead of fully moving the substrate to an aqueous environment, the transporter would shift the substrate to a location of intermediate hydrophobicity on the exoplasmic side of the protein. The substrate would be in an energetically unfavorable environment and would therefore be presented in an activated state to an acceptor [6,71]. Because the shift would not require a reorientation of the polar headgroup we term this "flopless activation". For the multidrug transporters, this model would imply that the relatively hydrophilic drugs would be immediately released from this site into the aqueous phase, turning this model into the hydrophobic vacuum cleaner model. In contrast, in the case of lipid transporters, the lipids would be stuck to the protein unless a hydrophobic acceptor surface would allow the lipid to leave. Transfer of the lipid from the exoplasmic site on the transporter to the acceptor would be most efficient if this acceptor would be bound directly to the transporter. In the absence of an acceptor, there are various possibilities depending on how the exoplasmic binding site connects to the exoplasmic bilayer leaflet. One extreme case would be that only the presence of an acceptor would allow substrate release. This would lead to the interesting possibility that the $\mathrm{ABC}$ transporter can only start a new reaction cycle in the presence of the acceptor. In the other extreme case, the exoplasmic binding site in the $\mathrm{ABC}$ transporter would open directly into the exoplasmic leaflet of the membrane. In this case the model becomes identical to the flippase model with the disadvantageous loss of coupling between the translocation within the protein to the transfer onto the acceptor. In the intermediate situation, the lipid is able to move from the transporter into the exoplasmic leaflet of the membrane but with lower probability than moving onto its cognate acceptor. The possibility that the transfer into the membrane is regulated by other (membrane) proteins cannot be excluded. In other words translocation could be uncoupled from the presence of the acceptor and the transporter would temporarily turn into a flippase. This suggests the intriguing possibility that while under normal circumstances the inward lipid flipping by P-type ATPases would promote vesicle budding into the cytosol $[10,11]$, under special conditions $A B C$ transporters could counteract this force and might even drive budding in the opposite direction. An inhibitory effect of ABCA1 on endocytosis has been observed [63,72].

Actually, the evidence that $\mathrm{ABC}$ transporters can flop lipid molecules from the cytosolic to the exoplasmic bilayer leaflet is remarkably scarce and indirect. (i) A floppase activity has been reported for lipid analogs with one or two shortened and often polar fatty acyl chains $[4,60-62,64-68,73]$. Such lipid analogs have a much reduced activation energy for desorption [74], and thus their flop is no proof for a flippase model: they could be extruded into the aqueous phase and partition back into the (exoplasmic surface of) the membrane. (ii) Some of the lipid analogs flopped by ABCB1 after reconstitution in model membranes contained two long-chain fatty acids like dipalmitoyl- and dioleoyl-PE labeled on the head group with the fluorescent marker NBD [67]. (iii) Erythrocytes from 
knock-out mice lacking Abcal expressed even less PS on their cell surface than normal $[56,63]$, suggesting that ABCA1 may flop natural PS. (iv) Radiolabeled PC was translocated from the inner to the outer leaflet of mouse erythrocytes by a process that was ATP-dependent, was inhibited by glibenclamide, and was reduced in knock-out mice for Abcb1 or Abcb4 [59], suggesting that these molecules flop PC in isolated erythrocytes.

\section{Mechanistic variations}

Similar to the mammalian ABC lipid transporters described above, the plant Cer5 ABC transporter was required for the transport of wax molecules ( $\geqslant \mathrm{C} 26$ ) across the plasma membrane of epithelial cells to the extracellular cuticle [75], and the bacterial ABC transporter MsbA has been found essential for the transport of lipid A from the cytosolic surface of the E. coli inner membrane across that membrane to the outer membrane [76]. However, many bacterial ABC transporters transport molecules in the opposite direction into the cytosol [77]. Invariably, the substrate is supplied to the exoplasmic face of the transporter by a specific binding protein. This is exactly the opposite of the export of lipid molecules by the mammalian ABC transporters, but involves the same molecular interactions (Fig. 4). Actually, also some mammalian ABC lipid transporters may transport towards the cytosol. The presence of intact $\mathrm{ABCC} 7$ has been correlated with an increased uptake of the signaling lipids sphingosine-1-phosphate and lysophosphatidic acid [78]. In addition, ABCA4 apparently transports $N$-retinylidene-PE towards the cytosolic side of the disc membrane, where it is converted back to all-trans-retinal [47]. It is unclear how $N$-retinylidene-PE enters the exoplasmic binding site. Access of a lipid to an ABC transporter from the exoplasmic surface has been shown for the bacterial ABC transporter LolCDE. It allows a lipid-anchored protein on the exoplasmic side of the $E$. coli inner membrane to enter the binding site and subsequently extrudes the lipoprotein onto an acceptor, the periplasmic carrier LolA [79]. In mammals, a similar mechanism may be responsible for transferring morphogens like Hedgehog, which is anchored to the membrane by cholesterol and palmitate tails, and glycosylphosphatidylinositol-anchored proteins from the cell surface to blood lipoproteins, where they have been found in Drosophila [80].

Although only little evidence is available on the substrate specificities of the various $\mathrm{ABC}$ transporters due to the lack of adequate methods, they are clearly diverse. This is well-illustrated by the ABCA subfamily. While ABCA1 and A7 are involved in transport of $\mathrm{PC}, \mathrm{SM}$ and cholesterol (cholesterol contested for A7 [81]), ABCA4 seems specific for $N$-retinylidene-PE, and the lamellar body transporters $\mathrm{ABCA} 3$ and ABCA12 may be specific for dipalmitoyl PC (possibly in combination with cholesterol) and glucosylceramide (plus cholesterol?), respectively (see above). Best characterized is the selectivity of human ABCB4 for PC: PC is the only phospholipid in human bile [82], while some other mammalian biles contain SM as well. A selectivity for the translocation of short-chain PCs over analogs of PE and sphingolipids was then observed in transfected cells [4] and in an in vitro system [66], although the latter study missed the fact that $\mathrm{ABCB} 1$ is an even better transporter for these analogs and displays essentially no selectivity [4]. ABCB1 was reported to also flip a long-chain fluorescent PE analog [67] and maybe cholesterol (discussed in [83]), and its presence correlated with an enhanced PS exposure on the cell surface [62]. Still, after numerous studies on the transport activity of $\mathrm{ABCB} 1$, one must conclude that it is primarily a multidrug transporter that can also transport the short-chain PC platelet activating factor [50,51] and that maybe, under certain conditions, flops natural long-chain lipids. In contrast, $\mathrm{ABCB} 4$ is primarily a $\mathrm{PC}$ transporter, that also pumps some drugs (see [84]). The multidrug transporter $\mathrm{ABCC} 1$, like ABCB1, has been reported to transport analogs of membrane lipids. However, no natural lipid substrates have been identified for the ABCC subfamily. The ABCD1-4 halftransporters homo- or heterodimerize and they probably bind and transport very long-chain fatty acids or their-SCoA esters $[43,44]$. In the ABCG family, the ABCG5/G8 heterodimer transports cholesterol into the intestinal lumen and into bile [29,30], while ABCG1 transports cholesterol to HDL [34]. The fact that also this transport to HDL is accompanied by transport of the choline-phospholipids suggests that cholesterol may be transported in a complex with PC or SM.

\section{Future perspectives}

The discovery that a number of inherited lipid-linked disorders are due to mutations in ABC transporters has attracted many research groups to the field. Clearly, the action of these transporters is fundamental to a number of aspects of human physiology, and an understanding of the basic principles of their action will greatly improve the diagnosis and treatment of lipid-related diseases in general. Considering the large number of $\mathrm{ABC}$ lipid transporters, mammals have developed many different applications for what seems to be one basic mechanism: transport of lipids out of the membrane onto some acceptor. It is a challenge to uncover the mechanistics of the transport and to understand the structural specificity of the transporters. For this it will be necessary to reconstitute the transporters in model membranes and rebuild their cellular context from chemically defined components to recreate their function. In the end, it will be necessary to describe their activities within the complex lipidomics of the human body $[85,86]$. This effort will require new methodological approaches and, above all, a lot of basic research.

Acknowledgements: We are grateful for insightful discussions with Winchil Vaz, University of Coimbra, Portugal. We thank EC Flippase network (MRTN-CT-2004-5330) and European Lipidomics Initiative (LSSG-CT-2004-013032) for stimulation and financial support.

\section{References}

[1] Smit, J.J. et al. (1993) Homozygous disruption of the murine mdr2 P-glycoprotein gene leads to a complete absence of phospholipid from bile and to liver disease. Cell 75, 451-462.

[2] Gottesman, M.M. and Ling, V. (2005) The molecular basis of multidrug resistance in cancer: The early years of P-glycoprotein research. FEBS Lett., this issue.

[3] Mosser, J. et al. (1993) Putative X-linked adrenoleukodystrophy gene shares unexpected homology with $\mathrm{ABC}$ transporters. Nature 361, 726-730.

[4] van Helvoort, A., Smith, A.J., Sprong, H., Fritzsche, I., Schinkel, A.H., Borst, P. and van Meer, G. (1996) MDR1 P-glycoprotein is a lipid translocase of broad specificity, while MDR3 P-glycoprotein specifically translocates phosphatidylcholine. Cell 87, 507517. 
[5] Borst, P., Zelcer, N. and van Helvoort, A. (2000) ABC transporters in lipid transport. Biochim. Biophys. Acta 1486, $128-144$.

[6] Pohl, A., Devaux, P.F. and Herrmann, A. (2005) Function of prokaryotic and eukaryotic $\mathrm{ABC}$ proteins in lipid transport. Biochim. Biophys. Acta 1733, 29-52.

[7] Holthuis, J.C. and Levine, T.P. (2005) Lipid traffic: floppy drives and a superhighway. Nat. Rev. Mol. Cell. Biol. 6, 209-220.

[8] Chang, Q.L., Gummadi, S.N. and Menon, A.K. (2004) Chemical modification identifies two populations of glycerophospholipid flippase in rat liver ER. Biochemistry 43, 10710-10718.

[9] Farge, E., Ojcius, D.M., Subtil, A. and Dautry-Varsat, A. (1999) Enhancement of endocytosis due to aminophospholipid transport across the plasma membrane of living cells. Am. J. Physiol. 276, C725-C733.

[10] Graham, T.R. (2004) Flippases and vesicle-mediated protein transport. Trends Cell. Biol. 14, 670-677.

[11] Pomorski, T., Holthuis, J.C., Herrmann, A. and van Meer, G. (2004) Tracking down lipid flippases and their biological functions. J. Cell Sci. 117, 805-813.

[12] Pohl, E.E., Peterson, U., Sun, J. and Pohl, P. (2000) Changes of intrinsic membrane potentials induced by flip-flop of long-chain fatty acids. Biochemistry 39, 1834-1839.

[13] Steck, T.L., Ye, J. and Lange, Y. (2002) Probing red cell membrane cholesterol movement with cyclodextrin. Biophys. J. $83,2118-2125$.

[14] Hamilton, J.A. (2003) Fast flip-flop of cholesterol and fatty acids in membranes: implications for membrane transport proteins. Curr. Opin. Lipidol. 14, 263-271.

[15] Bai, J. and Pagano, R.E. (1997) Measurement of spontaneous transfer and transbilayer movement of BODIPY-labeled lipids in lipid vesicles. Biochemistry 36, 8840-8848.

[16] López-Montero, I., Rodriguez, N., Cribier, S., Pohl, A., Velez, M. and Devaux, P.F. (2005) Rapid transbilayer movement of ceramides in phospholipid vesicles and in human erythrocytes. J. Biol. Chem. 280, 25811-25819.

[17] Sampaio, J.L., Moreno, M.J. and Vaz, W.L. (2005) Kinetics and thermodynamics of association of a fluorescent lysophospholipid derivative with lipid bilayers in liquid-ordered and liquid-disordered phases. Biophys. J. 88, 4064-4071.

[18] Abreu, M.S., Moreno, M.J. and Vaz, W.L. (2004) Kinetics and thermodynamics of association of a phospholipid derivative with lipid bilayers in liquid-disordered and liquid-ordered phases. Biophys. J. 87, 353-365.

[19] Phillips, M.C., Johnson, W.J. and Rothblat, G.H. (1987) Mechanism and consequences of cellular cholesterol exchange and transfer. Biochim. Biophys. Acta 906, 223-276.

[20] Jones, J.D. and Thompson, T.E. (1990) Mechanism of spontaneous, concentration-dependent phospholipid transfer between bilayers. Biochemistry 29, 1593-1600.

[21] Baumann, N.A., Sullivan, D.P., Ohvo-Rekila, H., Simonot, C., Pottekat, A., Klaassen, Z., Beh, C.T. and Menon, A.K. (2005) Transport of newly synthesized sterol to the sterolenriched plasma membrane occurs via nonvesicular equilibration. Biochemistry 44, $5816-5826$

[22] Hanada, K., Kumagai, K., Yasuda, S., Miura, Y., Kawano, M., Fukasawa, M. and Nishijima, M. (2003) Molecular machinery for non-vesicular trafficking of ceramide. Nature 426, 803-809.

[23] Mogelsvang, S., Marsh, B.J., Ladinsky, M.S. and Howell, K.E. (2004) Predicting function from structure: 3D structure studies of the mammalian Golgi complex. Traffic 5, 338-345.

[24] Deleuze, J.F., Jacquemin, E., Dubuisson, C., Cresteil, D., Dumont, M., Erlinger, S., Bernard, O. and Hadchouel, M (1996) Defect of multidrug-resistance 3 gene expression in a subtype of progressive familial intrahepatic cholestasis. Hepatology 23, 904-908.

[25] de Vree, J.M. et al. (1998) Mutations in the MDR3 gene cause progressive familial intrahepatic cholestasis. Proc. Natl. Acad Sci. USA 95, 282-287.

[26] Ehehalt, R., Jochims, C., Lehmann, W.D., Erben, G., Staffer, S., Reininger, C. and Stremmel, W. (2004) Evidence of luminal phosphatidylcholine secretion in rat ileum. Biochim. Biophys. Acta 1682, 63-71.
[27] Gerloff, T., Stieger, B., Hagenbuch, B., Madon, J., Landmann, L., Roth, J., Hofmann, A.F. and Meier, P.J. (1998) The sister of Pglycoprotein represents the canalicular bile salt export pump of mammalian liver. J. Biol. Chem. 273, 10046-10050.

[28] Strautnieks, S.S. et al. (1998) A gene encoding a liver-specific $\mathrm{ABC}$ transporter is mutated in progressive familial intrahepatic cholestas is. Nat. Genet. 20, 233-238.

[29] Langheim, S., Yu, L., von Bergmann, K., Lutjohann, D., Xu, F., Hobbs, H.H. and Cohen, J.C. (2005) ABCG5 and ABCG8 require MDR2 for secretion of cholesterol into bile. J. Lipid Res. 46, 1732-1738

[30] Berge, K.E. et al. (2000) Accumulation of dietary cholesterol in sitosterolemia caused by mutations in adjacent $\mathrm{ABC}$ transporters. Science 290, 1771-1775.

[31] Rust, S. et al. (1999) Tangier disease is caused by mutations in the gene encoding ATP-binding cassette transporter 1. Nat. Genet. 22, 352-355.

[32] Brooks-Wilson, A. et al. (1999) Mutations in $A B C 1$ in Tangier disease and familial high-density lipoprotein deficiency. Nat. Genet. 22, 336-345.

[33] Bodzioch, M. et al. (1999) The gene encoding ATP-binding cassette transporter 1 is mutated in Tangier disease. Nat. Genet. $22,347-351$.

[34] Klucken, J. et al. (2000) ABCG1 (ABC8), the human homolog of the Drosophila white gene, is a regulator of macrophage cholesterol and phospholipid transport. Proc. Natl. Acad. Sci. USA 97, 817-822.

[35] Wang, N., Lan, D., Chen, W., Matsuura, F. and Tall, A.R. (2004) ATP-binding cassette transporters G1 and G4 mediate cellular cholesterol efflux to high-density lipoproteins. Proc. Natl. Acad. Sci. USA 101, 9774-9779.

[36] Yamano, G. et al. (2001) ABCA3 is a lamellar body membrane protein in human lung alveolar type II cells. FEBS Lett. 508, 221225 .

[37] Shulenin, S., Nogee, L.M., Annilo, T., Wert, S.E., Whitsett, J.A. and Dean, M. (2004) ABCA3 gene mutations in newborns with fatal surfactant deficiency. N. Engl. J. Med. 350, 1296-1303.

[38] Nagata, K., Yamamoto, A., Ban, N., Tanaka, A.R., Matsuo, M., Kioka, N., Inagaki, N. and Ueda, K. (2004) Human ABCA3, a product of a responsible gene for abca3 for fatal surfactant deficiency in newborns, exhibits unique ATP hydrolysis activity and generates intracellular multilamellar vesicles. Biochem. Biophys. Res. Commun. 324, 262-268.

[39] Lefevre, C. et al. (2003) Mutations in the transporter ABCA12 are associated with lamellar ichthyosis type 2 . Hum. Mol. Genet. $12,2369-2378$.

[40] Akiyama, M. et al. (2005) Mutations in lipid transporter ABCA12 in harlequin ichthyosis and functional recovery by corrective gene transfer. J. Clin. Invest. 115, 1777-1784.

[41] Kelsell, D.P. et al. (2005) Mutations in ABCA12 underlie the severe congenital skin disease harlequin ichthyosis. Am. J. Human Genet. 76, 794-803.

[42] Elias, P.M., Fartasch, M., Crumrine, D., Behne, M., Uchida, Y. and Holleran, W.M. (2000) Origin of the corneocyte lipid envelope (CLE): observations in harlequin ichthyosis and cultured human keratinocytes. J. Invest. Dermatol. 115, 765-769.

[43] Ferrer, I. et al. (2005) Inactivation of the peroxisomal ABCD2 transporter in the mouse leads to late-onset ataxia involving mitochondria, Golgi and endoplasmic reticulum. Hum. Mol. Genet. 23, 3565-3577.

[44] Guimaraes, C.P., Sa-Miranda, C. and Azevedo, J.E. (2005) Probing substrate-induced conformational alterations in adrenoleukodystrophy protein by proteolysis. J. Human Genet. 50, 99 105 .

[45] Weng, J., Mata, N.L., Azarian, S.M., Tzekov, R.T., Birch, D.G. and Travis, G.H. (1999) Insights into the function of Rim protein in photoreceptors and etiology of Stargardt's disease from the phenotype in abcr knockout mice. Cell 98, 13-23.

[46] Beharry, S., Zhong, M. and Molday, R.S. (2004) $N$-retinylidenephosphatidylethanolamine is the preferred retinoid substrate for the photoreceptor-specific ABC transporter ABCA4 (ABCR). J. Biol. Chem. 279, 53972-53979.

[47] Strauss, O. (2005) The retinal pigment epithelium in visual function. Physiol. Rev. 85, 845-881. 
[48] Borst, P., Zelcer, N., van de Wetering, K. and Poolman, B. (2005) On the putative co-transport of drugs by multidrug resistance proteins. FEBS Lett., this issue, doi:10.1016/j.febslet.2005.12.039.

[49] Deeley, R.G. and Cole, S.P.C. (2005) Substrate recognition and transport by multidrug resistance protein (MRP)1 (ABCC1). FEBS Lett., this issue, doi:10.1016/j.febslet.2005.12.036.

[50] Ernest, S. and Bello-Reuss, E. (1999) Secretion of plateletactivating factor is mediated by MDR1 P- glycoprotein in cultured human mesangial cells. J. Am. Soc. Nephrol. 10, 2306 2313.

[51] Raggers, R.J., Vogels, I. and van Meer, G. (2001) Multidrugresistance P-glycoprotein (MDR1) secretes platelet-activating factor. Biochem. J. 357, 859-865.

[52] Gordon, D.A. and Jamil, H. (2000) Progress towards understanding the role of microsomal triglyceride transfer protein in apolipoprotein-B lipoprotein assembly. Biochim. Biophys. Acta $1486,72-83$.

[53] Winau, F. et al. (2004) Saposin C is required for lipid presentation by human CD1b. Nat. Immunol. 5, 169-174.

[54] Smith, A.J., Timmermans-Hereijgers, J.L.P.M., Roelofsen, B., Wirtz, K.W.A., van Blitterswijk, W.J., Smit, J.J.M., Schinkel, A.H. and Borst, P. (1994) The human MDR3 Pglycoprotein promotes translocation of phosphatidylcholine through the plasma membrane of fibroblasts from transgenic mice. FEBS Lett. 354, 263-266.

[55] Fitscher, B.A., Ehehalt, R., Jochims, C., Pohl, J., Herrmann, T. and Stremmel, W. (2000) Functional and histochemical analysis of MDR3 P-glycoprotein in a tetracycline-controlled gene expression system. Eur. J. Med. Res. 5, 517-522.

[56] Hamon, Y. et al. (2000) ABC1 promotes engulfment of apoptotic cells and transbilayer redistribution of phosphatidylserine. Nat. Cell Biol. 2, 399-406.

[57] Albrecht, C. et al. (2005) A novel missense mutation in ABCA1 results in altered protein trafficking and reduced phosphatidylserine translocation in a patient with Scott syndrome. Blood 106, 542-549.

[58] Renooij, W., Van Golde, L.M., Zwaal, R.F. and van Deenen, L.L. (1976) Topological asymmetry of phospholipid metabolism in rat erythrocyte membranes. Evidence for flip-flop of lecithin. Eur. J. Biochem. 61, 53-58.

[59] Kälin, N., Fernandes, J., Hrafnsdottir, S. and van Meer, G. (2004) Natural phosphatidylcholine is actively translocated across the plasma membrane to the surface of mammalian cells. J. Biol. Chem. 279, 33228-33236.

[60] Raggers, R.J., van Helvoort, A., Evers, R. and van Meer, G. (1999) The human multidrug resistance protein MRP1 translocates sphingolipid analogs across the plasma membrane. J. Cell Sci. 112, 415-422.

[61] Bosch, I., Dunussi-Joannopoulos, K., Wu, R.-L., Furlong, S.T. and Croop, J. (1997) Phosphatidylcholine and phosphatidylethanolamine behave as substrates of the human MDR1 P-glycoprotein. Biochemistry 36, 5685-5694.

[62] Pohl, A., Lage, H., Muller, P., Pomorski, T. and Herrmann, A. (2002) Transport of phosphatidylserine via MDR1 (multidrug resistance 1)P-glycoprotein in a human gastric carcinoma cell line. Biochem. J. 365, 259-268.

[63] Alder-Baerens, N., Muller, P., Pohl, A., Korte, T., Hamon, Y., Chimini, G., Pomorski, T. and Herrmann, A. (2005) Headgroupspecific exposure of phospholipids in ABCA1-expressing cells. J. Biol. Chem. 280, 26321-26329.

[64] Dekkers, D.W., Comfurius, P., Schroit, A.J., Bevers, E.M. and Zwaal, R.F. (1998) Transbilayer movement of NBD-labeled phospholipids in red blood cell membranes: outward-directed transport by the multidrug resistance protein 1 (MRP1). Biochemistry 37, 14833-14837.

[65] Kamp, D. and Haest, C.W.M. (1998) Evidence for a role of the multidrug resistance protein (MRP) in the outward translocation of NBD-phospholipids in the erythrocyte membrane. Biochim. Biophys. Acta 1372, 91-101.

[66] Ruetz, S. and Gros, P. (1994) Phosphatidylcholine translocase: a physiological role for the $m d r 2$ gene. Cell 77, 1-20

[67] Romsicki, Y. and Sharom, F.J. (2001) Phospholipid flippase activity of the reconstituted p-glycoprotein multidrug transporter. Biochemistry 40, 6937-6947.
[68] Eckford, P.D. and Sharom, F.J. (2005) The reconstituted Pglycoprotein multidrug transporter is a flippase for glucosylceramide and other simple glycosphingolipids. Biochem. J. 389, 517-526.

[69] Higgins, C.F. and Gottesman, M.M. (1992) Is the multidrug transporter a flippase? Trends Biochem. Sci. 17, 18-21.

[70] Raviv, Y., Pollard, H.B., Bruggemann, E.P., Pastan, I. and Gottesman, M.M. (1990) Photosensitized labeling of a functional multidrug transporter in living drug-resistant tumor cells. J. Biol. Chem. 265, 3975-3980.

[71] Small, D.M. (2003) Role of ABC transporters in secretion of cholesterol from liver into bile. Proc. Natl. Acad. Sci. USA 100, 4-6.

[72] Zha, X., Genest Jr., J. and McPherson, R. (2001) Endocytosis is enhanced in Tangier fibroblasts. Possible role of ATP-binding cassette protein A1 in endosomal vesicular transport. J. Biol. Chem. 276, 39476-39483.

[73] van Helvoort, A., Giudici, M.L., Thielemans, M. and van Meer, G. (1997) Transport of sphingomyelin to the cell surface is inhibited by brefeldin $\mathrm{A}$ and in mitosis, where $\mathrm{C}_{6}$-NBD-sphingomyelin is translocated across the plasma membrane by a multidrug transporter activity. J. Cell Sci. 110, 75-83.

[74] Nichols, J.W. (1985) Thermodynamics and kinetics of phospholipid monomer-vesicle interaction. Biochemistry 24, 6390 6398

[75] Pighin, J.A., Zheng, H., Balakshin, L.J., Goodman, I.P., Western, T.L., Jetter, R., Kunst, L. and Samuels, A.L. (2004) Plant cuticular lipid export requires an ABC transporter. Science 306, 702-704.

[76] Doerrler, W.T., Gibbons, H.S. and Raetz, C.R. (2004) MsbAdependent translocation of lipids across the inner membrane of Escherichia coli. J. Biol. Chem. 279, 45102-45109.

[77] Biemans-Oldehinkel, E., Doeven, M.K. and Poolman, B. (2005) $\mathrm{ABC}$ transporter architecture and regulatory roles of accessory domains. FEBS Lett., this issue, doi:10.1016/j.febslet.2005.11.079.

[78] Boujaoude, L.C., Bradshaw-Wilder, C., Mao, C., Cohn, J., Ogretmen, B., Hannun, Y.A. and Obeid, L.M. (2001) Cystic fibrosis transmembrane regulator regulates uptake of sphingoid base phosphates and lysophosphatidic acid: modulation of cellular activity of sphingosine 1-phosphate. J. Biol. Chem. 276, 35258-35264.

[79] Narita, S.-i. and Tokuda, H. (2005) An ABC transporter mediating the membrane detachment of bacterial lipoproteins depending on their sorting signals. FEBS Lett., this issue, doi:10.1016/j.febslet.2005.10.038.

[80] Panáková, D., Sprong, H., Marois, E., Thiele, C. and Eaton, S (2005) Lipoprotein particles are required for Hedgehog and Wingless signalling. Nature 435, 58-65.

[81] Abe-Dohmae, S., Ueda, K. and Yokoyama, S. (2005) ABCA7, a molecule with unknown function. FEBS Lett., this issue, doi:10.1016/j.febslet.2005.12.029.

[82] Moschetta, A. et al. (2005) A phylogenetic survey of biliary lipids in vertebrates. J. Lipid Res. 46, 2221-2232.

[83] Le Goff, W., Settle, M., Greene, D.J., Morton, R.E. and Smith, J.D. (2005) Reevaluation of the role of the multidrug resistant Pglycoprotein in cellular cholesterol homeostasis. J. Lipid Res., in press.

[84] Smith, A.J. et al. (2000) MDR3 P-glycoprotein, a phosphatidylcholine translocase, transports several cytotoxic drugs and directly interacts with drugs as judged by interference with nucleotide trapping. J. Biol. Chem. 275, 23530-23539.

[85] van Meer, G. (2005) Cellular lipidomics. EMBO J. 24, 3159-3165.

[86] Wenk, M.R. (2005) The emerging field of lipidomics. Nat. Rev. Drug Discov. 4, 594-610.

[87] Yancey, P.G., Rodrigueza, W.V., Kilsdonk, E.P.C., Stoudt, G.W., Johnson, W.J., Phillips, M.C. and Rothblat, G.H. (1996) Cellular cholesterol efflux mediated by cyclodextrins. Demonstration of kinetic pools and mechanism of efflux. J. Biol. Chem. 271, $16026-16034$

[88] Parsegian, V.A., Fuller, N. and Rand, R.P. (1979) Measured work of deformation and repulsion of lecithin bilayers. Proc. Natl Acad. Sci. USA 76, 2750-2754.

[89] Almeida, P.F. (1999) Lipid transfer between vesicles: effect of high vesicle concentration. Biophys. J. 76, 1922-1928. 